\title{
RESEÑA DEL LIBRO: APUNTES SOBRE CIENCIA E INVESTIGACIÓN CIENTÍFICA EN REPÚBLICA DOMINICANA. GÉNESIS Y EVOLUCIÓN DEL FONDO NACIONAL DE INNOVACIÓN Y DESARROLLO CIENTÍFICO Y TECNOLÓGICO (FONDOCYT), 1998-2018
}

\begin{abstract}
Book Review: Notes on Science and Scientific Research in the Dominican Republic. Genesis and Evolution of the National Fund for Innovation and Scientific and Technological Development (FONDOCYT), 1998-2018
\end{abstract}

\section{Julia Matilde Mota}

Escuela de Biología. Facultad de Ciencias Universidad Autónoma de Santo Domingo (UASD), Santo Domingo. República Dominicana. ORCID: 0000-0002-6045-5184, Correo-e: jmota64@uasd.edu.do

Recibido: 6-XI-2020 • Aprobado: 2-XII-2020

Cómo citar: Mota, J. M. (2020). Reseña del libro: Apuntes sobre Ciencia e Investigación Científica en República Dominicana. Génesis y Evolución del Fondo Nacional de Innovación y Desarrollo Científico y Tecnológico (FONDOCYT), 1998-2018. Ciencia, Ambiente y Clima, 3(2), 59-61. Doi: https://doi.org/10.22206/cac.2020.v3i2.pp59-61

El pasado miércoles 12 de agosto del ańo en curso, fue puesto en circulación virtual el libro "Apuntes sobre Ciencia e Investigación Cientifica en República Dominicana. Génesis y Evolución del Fondo Nacional de Innovación y Desarrollo Cientifico y Tecnológico (FONDOCYT), 1998-2018" de la autoría de los investigadores Placido Gómez, Sixto Incháustegui y Carlos Rodríguez.

El libro es una vasta obra de 383 páginas dividida en 7 capítulos, donde se realiza una retrospectiva del desarrollo de las investigaciones científicas en el país. En la parte II, aparecen los resúmenes, investigadores y montos los proyectos aprobados, desde el año 2005.

En el primer capítulo, se reconoce el esfuerzo del país para crear capacidades entre el desarrollo económico y científico- tecnológico y las fuentes de financiación por parte del Ministerio de Educación Superior Ciencia y Tecnología (MESCYT) a través del Fondo Nacional de Innovación y Desarrollo Científico y Tecnológico (FONDOCYT), entre los años 1998 a 2018. Este fondo de financiamiento, entró en vigencia en el año 2005.

En sus inicios, el documento recopila los aportes científicos llevado a cabo entre los siglos XV al XX por parte de investigadores nacionales y extranjeros, sus nombres y área de investigación, entre las cuales sobresalen los trabajos realizados en el área de botánica. También se resalta el incremento a partir del año 1961, de proyectos de investigación realizados por unas 50 instituciones, entre las cuales figuran universidades e institutos tecnológicos y especializados.

La obra hace mención de un Plan Estratégico de Ciencia, Tecnología e Innovación 2008-2018 (PECYTI), cuyo objetivo según la obra "es la de producir desarrollo científico y tecnológico significativo, y que sirva de catalítico a innovaciones empresariales." Expresan, que el plan consta de cuatro objetivos estratégicos con diferentes políticas y programas para su implementación. Menciona, además, que pretenden su implementación con la sinergia de múltiples instituciones, haciendo 
mención de instituciones estatales involucradas en el quehacer científico e insta a otros ministerios e instituciones gubernamentales a jugar su rol.

Como logros, se destaca el incremento de la financiación de proyectos a partir del año 2010. En los mismos, además del importe de FONDOCYT, se grafica la contrapartida de las instituciones involucradas.

En el periodo 2005-2018, la mayor cantidad de proyectos aprobados (133 proyectos), versaban sobre sobre Biotecnología y Recursos genéticos, producción sostenible y seguridad alimentaria, encontrándose el menor número en investigaciones sobre Medio Ambiente y Recursos Naturales.

Respecto al número de investigaciones realizadas, se muestra un directorio creado por el Instituto Dominicano de Tecnología (INDOTEC) en el año 1984, donde reporta unas 319 investigaciones con una mayor concentración en proyectos del área de Ciencias de la salud, reflejando una escasez en las ciencias fundamentales.

Con relación a lo anterior, se resalta la importancia de las Ciencias Básicas como eje fundamental para el desarrollo científico y nos ofrece una descripción de los logros obtenidos en investigaciones nacionales, dentro de los campos de Agricultura, Biotecnologías, las Ingenierías y Energías Renovables.

Respecto a las Ciencias Básicas, entre los años 20052018 , fueron propuestos 118 proyectos, recayendo un $81 \%$ en Institutos de Educación Superior, con una mayor concentración en la Universidad Autónoma de Santo Domingo, seguidos por la Universidad Tecnológica de Santo Domingo (INTEC) y la Pontificia Universidad Católica y Maestra (PUCMM) entre otras. En cuanto a los centros de investigaciones apenas representaron un 19\%.

En el documento, también aparecen reflejados los proyectos aprobados en las áreas de Salud y Biomédica (73 proyectos) y en el área de Desa- rrollo de Software, mecatrónica, servicios y transporte ( 24 proyectos)

En el capítulo IV referente a Biotecnología, Recursos genéticos, Producción sostenible y Seguridad alimentaria, aparece una propuesta para la conformación de un consorcio científico - tecnológico para el desarrollo de la República dominicana.

Entre los logros, se enfatiza que se han cuadriplicado el número de publicaciones de autores dominicanos, por lo cual se incentiva a los investigadores a seguir publicando sus resultados, a generar patentes y a una reflexión sobre la necesidad de crear las infraestructuras necesarias con el instrumental de laboratorio requerido y el personal científico-técnico de calidad. Por lo cual consideramos propicio, que el Ministerio de Ciencia y Tecnología de la República Dominicana en conjunto con los institutos de investigaciones de la academia y de los Ministerios con Laboratorios de Investigación, establezcan un plan de planificación coordinado dirigido a la ejecución de proyectos de investigación-desarrollo enfocados a la necesidad país.

En el capítulo V se basa en un análisis de las investigaciones realizadas en salud y biomedicina enfocándose en las siguientes áreas: medicina, enfermería, ciencias farmacéuticas, bioanálisis y odontología.

Continuando con el capítulo VI esta enfocado en medio ambiente y los recursos naturales y las investigaciones que se han realizado a partir del 2005, donde se establecen diferencias entre los proyectos realizados por hombres y mujeres y ofrece datos de las instituciones que han recibido financiamiento y los impactos futuros que se esperan.

Concluyendo con el capítulo VII hace una revisión sobre proyectos sobre el desarrollo de software, mecatrónica, servicios y transporte, ingeniería, energía y biocombustibles, indica que esta gama de investigaciones se inició en el 2005, con una financiación de 57 proyectos. 
Reseña del libro: Apuntes sobre Ciencia e Investigación Científica en República Dominicana. Génesis y Evolución del Fondo Nacional de Innovación y Desarrollo Científico y Tecnológico (FONDOCYT), 1998-2018

Finalmente consideramos, que de acuerdo con los resultados podemos concluir, que la sociedad científica dominicana debe reconocer el rol de financiación de FONDOCYT para el desarrollo de proyectos de investigación a nivel nacional para de ese modo contribuir con el desarrollo económico y científico- tecnológico para el beneficio del país. 